\title{
Analysis of Biological Pathways Involved in the Pathogenesis of Bipolar Disorder
}

\author{
Mamdouh, Deeksha Yelamanchi, Arya Nalavade, Aliza Lopez \\ DOI: 10.29322/IJSRP.11.02.2021.p11052 \\ http://dx.doi.org/10.29322/IJSRP.11.02.2021.p11052
}

Varshene Sivaprakash, Ulain Umar, Trisha Thontepu, Rehan Shah, Karina Anders, Jennifer Xu, Jaylen Lockhart, Gasser

\begin{abstract}
Bipolar disorder (BD) is a mental health condition that affects approximately $1 \%$ of the global population. It is characterized by extreme euphoric mood swings and depressive and manic episodes. Despite the increasing number of patients world-wide, there are very few viable treatments that have proven efficacy for treating bipolar disorder. A challenge for the development of novel treatment options, is a relative lack of understanding into the causes and biological mechanisms driving BD onset. Recent research has consistently found a high degree of heritability of $\mathrm{BD}$, with estimates between $60 \%$ to $80 \%$ and many genes playing a factor in the development of the disorder [1][7]. To better define genes and pathways that could potentially play a role in BD onset, we completed an analysis of human genetic data. Through the EMBL-EBI GWAS catalog, we analyzed the top 10 SNPs and genes with the strongest connection to BD. Using the EMBL-EBI GWAS Catalog to determine a list of genes significant to the cause of BD, a pathway analysis was conducted using the Database for Annotation, Visualization and Integrated Discovery (DAVID) to find the most significant pathways. As a result, it was found that alternative splicing is the most significant pathway in Bipolar disorder, alongside phosphoprotein, postsynaptic density, and immunoglobulin I-set. Multiple approaches of targeting bipolar disorder through these pathways were highlighted like usage of alternatively spliced genes as novel biomarkers. Thus, these pathways could be novel potential therapeutic targets for bipolar disorder in the future.
\end{abstract}

Index Terms- Bipolar disorder, Genetic Pathways, Single nucleotide polymorphisms

\section{INTRODUCTION}

Bipolar disorder, previously known as manic depressive disorder, is a chronic psychiatric disorder, which is typically characterized with episodes of mania, hypomania, and alternating episodes of depression [5]. While mania means the state of excessive energy, associated with euphoria, impulsivity, and psychosis, depression leads to non-reactive mood, disruption of sleep, eating routines, feelings of guilt and worthlessness. As of the latest estimates, nearly $1-2 \%$ of the world population is affected by bipolar disorder [2]. Despite the significant healthcare burden, there are only limited treatments available for bipolar disorder.

Here, we expand on previous research to characterize both known and novel biological pathways involved in the pathogenesis of BD. Using the EMBL-EBI GWAS catalog, we analyzed more than 1200 single nucleotide polymorphisms (SNPs) that have previously been associated with BD. Based on nearest genes, we next performed a pathway analysis using the Database for Annotation, Visualization and Integrated Discovery (DAVID) [4]. We found that individual SNPs for the nearest genes within the pathways caused a dysregulation that was linked with Bipolar Disorder. This research shows the linkage between the dysregulation caused by SNPs and Bipolar Disorder, and highlights both known and novel genes and pathways that should be further investigated for their role(s) in BD.

\section{IDENTIFY, RESEARCH AND COLLECT IDEA}

Prior research suggests that there is no single factor or gene that causes BD but rather a multitude of factors that contribute to the pathogenesis of bipolar disorder (National Institute of Mental Health, 2018). Research has shown that BD is a heritable disease, with a concordance rate for identical twins averaging $67 \%$ and heritability estimates of $85 \%$ [1][7]. Heritability of BD, combined with environmental factors, including trauma or stress, have contributed to the prevalence of BD [12]. Because of this genetic contribution towards BD risk, researchers have devoted the last 25 years to the search for specific genetic factors that may influence disease development.

Previous meta-analysis of pathways has shown that BD is highly correlated with glutamate receptor signaling, PLC signaling, CRH signaling, endothelin 1 signaling, cardiac beta-adrenergic signaling and cardiac hypertrophy signaling. Earlier examples cite the $\mathrm{PKC}$ and glycogen synthase kinase $3 \beta$ (GSK3 $\beta$ ) signaling pathways as correlated with BD [9]. 


\section{WRITE DOWN YOUR STUDIES AND FINDINGS}

\section{A. Bits and Pieces together}

\begin{tabular}{|c|c|c|c|c|c|c|c|}
\hline SNP & P Value & $\begin{array}{l}\text { Ranking } \\
\text { of Risk }\end{array}$ & Scores & Peaks & Gene & Function of Gene & Reported Trait(s) \\
\hline rs 13217619 & $1 \times 10-27$ & 5 & 0.58955 & 5 & ZSCAN31 & $\begin{array}{l}\text { Responsible for nucleic acid binding and DNA-binding transcription } \\
\text { factor activity }\end{array}$ & Schizophrenia, major depressive disorder, anorexia nervocsa, OCD, Bipolar Disorder \\
\hline rs1516725 & $1 \times 10-24$ & 5 & 0.70952 & 4 & ETV5 & $\begin{array}{l}\text { Responsible for DNA-binding transcription factor activity and } \\
\text { transcription regulatory region DNA binding }\end{array}$ & BMI, hip/waist circumference in men and women \\
\hline rs 3888190 & $1 \times 10-24$ & If & 0.55436 & 28 & ATP2A1 & $\begin{array}{l}\text { Responsible for calcium ion binding and nucleotide binding; instructions } \\
\text { for the SERCAl enzyme, regulating levels of calcium ions }\end{array}$ & BMI, specifically in relation to smoking \\
\hline rss12552 & $1 \times 10-23$ & 5 & 0.14427 & 3 & OLFM4 & $\begin{array}{l}\text { Responsible for protein homodimerization activity and cadherin binding; } \\
\text { facilitates cell adhesion }\end{array}$ & Bipolar Disorder, autism, MDD, intelligence \\
\hline rs 34706883 & $7 \times 10-20$ & $3 a$ & 0.47482 & 1078 & H2BC15 & $\begin{array}{l}\text { Responsible for transcription regulation, DNA repair, DNA replication } \\
\text { and chromosomal stability }\end{array}$ & Schizophrenia, Bipolar Disorder \\
\hline $\mathrm{r} 54298967$ & $8 \times 10-20$ & 5 & 0.05 & 18 & CACNAIC & $\begin{array}{l}\text { Regulates intracellular processes such as contraction, secretion, } \\
\text { neurotransmission and gene expression; instructions for calcium } \\
\text { channels and electrical signals }\end{array}$ & $\begin{array}{l}\text { anorexia nervosa, attention-deficithyperactivity disorder, autism spectrum disorder, bipolar } \\
\text { disorder, major depression, obsessive-compulsive disorder, schizophrenia, or Tourette } \\
\text { syndrome (pleiotropy) }\end{array}$ \\
\hline rs 151181 & $3 \times 10-19$ & If & 0.24002 & 14 & CLN3 & $\begin{array}{l}\text { Mediates microtubule-dependent, anterograde transport connecting the } \\
\text { Golgi network, endosomes, autophagosomes, lysosomes and plasma }\end{array}$ & $\begin{array}{l}\text { Core binding factor acute myeloid leukemia, bipolar disorder or body mass index, Crohn's } \\
\text { disease }\end{array}$ \\
\hline rs 75782365 & $3 \times 10-18$ & 5 & 1 & 6 & BTN3AI & Plays a role in T-cell activation and in the adaptive immune response. & $\begin{array}{l}\text { Autism spectrum disorder or schizophrenia, depression (broad), Help-seeking from a GP, } \\
\text { bipolar disorder (MTAG), schizophrenia (MTAG) }\end{array}$ \\
\hline rs4481150 & $5 \times 10-17$ & If & 0.11 & 21 & ITIH3 & $\begin{array}{l}\text { Stabilizes the extracellular matrix through its ability to bind hyaluronic } \\
\text { acid }\end{array}$ & $\begin{array}{l}\text { anorexia nervosa, attention-deficithyperactivity disorder, autism spectrum disorder, bipolar } \\
\text { disorder, major depression, obsessive-compulsive disorder, schizophrenia, or Tourette } \\
\text { syndrome (pleiotropy), Serum 25-Hydroxyvitamin D levels, red blood cell count }\end{array}$ \\
\hline rs4298967 & $3 \times 10-16$ & 5 & 0.05 & 18 & CACNAIC & $\begin{array}{l}\text { Regulates intracellular processes such as contraction, secretion, } \\
\text { neurotransmission and gene expression. }\end{array}$ & $\begin{array}{l}\text { anorexia nervosa, attention-deficit/ hyperactivity disorder, autism spectrum disorder, bipolar } \\
\text { disorder, major depression, obsessive-compulsive disorder, schizophrenia, or Tourette } \\
\text { syndrome (pleiotropy) }\end{array}$ \\
\hline
\end{tabular}

Figure 1

To begin to understand how genetic factors may influence biological mechanisms leading to BD, we started by analyzing the full repertoire of SNPs that had previously been associated with the disease. We used the EMBL-EBI GWAS catalog [6] to identify SNPs and their nearest genes that had previously reached genome-wide significance in 104 genome wide association studies. Approximately 1,200 SNPs were identified. The top SNPs with the most significant p-values are represented in Figure 1, with rs13217619, rs1516725, and rs3888190 among the top 3. Rs13217619, has been linked with a wide variety of mental disorders, including Schizophrenia, bipolar disorder, and Obsessive-Compulsive Disorder. rs1516725 and rs3888190, however, have both been associated with body mass index. Rs151181 is linked to bipolar disorder as well as Crohn's disease and rs4481150 is linked with multiple mental disorders, including bipolar disorder [6].

Since the top SNPs are in non-coding regions of the genome, we next asked if any SNPs might increase risk for BD by acting via gene regulatory element. We utilized RegulomeDB [3] which predicts the likelihood of genetic variants falling within and interrupting known or predicted enhancer elements. Each SNP receives a rank and a probability score from 0 to 1 based on the number of published genome-wide ChIP-seq peaks that the SNPs lie within in multiple different cell lines (Figure 2). The majority of our lead BD-associated SNPs have low RegulomeDB rank scores, signaling a high probability that they fall within and may interrupt gene regulation. SNPs rs 3888190 , rs 151181 , and rs 4481150 have rankings of 1f, which is the RegulomeDB score computation based on multiple high throughput datasets [3] (Figure 1).

In order to better understand the genes that are nearest to the BD-associated SNPs. By analyzing the functions of those genes and the biological pathways that they act within, we reasoned that we could identify known and novel mechanisms that may lead to disease. We utilized the Database for Annotation, Visualization and Integrated Discovery (DAVID) [4] to derive a list of pathways related to approximately 1250 genes nearest the BD-associated SNPs (Figure 2).

\begin{tabular}{|c|c|c|c|c|}
\hline Pathway Name & P Value & Number of BD Associated Genes in the Pathway & Percentage of BD Associated Genes in the Pathway & Category \\
\hline Alternative Splicing & 6.20E-21 & 411 & $0.50 \%$ & UP_KEYWORDS \\
\hline Splice Variant & $2.80 \mathrm{E}-20$ & 334 & $0.40 \%$ & UP_SEQ_FEAUTURE \\
\hline Phosphoprotein & $6.10 \mathrm{E}-12$ & 316 & $0.30 \%$ & UP_KEYWORDS \\
\hline Postsynaptic Density & 3.30E-08 & 23 & $0.00 \%$ & GOTERM_CC_dIRECT \\
\hline Immunoglobulin I-Set & 4.10E-08 & 20 & $0.00 \%$ & INTERPRO \\
\hline Dendritic Spine & 4.20E-08 & 17 & $0.00 \%$ & GOTERM_CC_dIRECT \\
\hline Calcium Signaling Pathway & $1.30 \mathrm{E}-06$ & 20 & $0.00 \%$ & KEGG_PATHWAY \\
\hline Immunoglobulin subtype 2 & $1.80 \mathrm{E}-06$ & 24 & $0.00 \%$ & INTERPRO \\
\hline Intracellular Signal Transduction & $4.70 \mathrm{E}-06$ & 31 & $0.00 \%$ & GOTERM_BP_DIRECT \\
\hline Calcium & 5.50E-06 & 50 & $0.10 \%$ & UP_KEYWORDS \\
\hline Membrane & $6.90 \mathrm{E}-06$ & 265 & $0.30 \%$ & UP_KEYWORDS \\
\hline Polymorphism & 7.70E-06 & 394 & $0.40 \%$ & UP_KEYWORDS \\
\hline Coiled Coil & $8.30 \mathrm{E}-06$ & 126 & $0.10 \%$ & UP_KEYWORDS \\
\hline Magnesium Ion Homeostasis & $1.10 \mathrm{E}-05$ & 5 & $0.00 \%$ & GOTERM_BP_DIRECT \\
\hline
\end{tabular}

Figure 2

B. Use of Simulation software

\section{EMBL-EBI GWAS-based SNP detection}

The EMBL-EBI GWAS Catalog [6], a catalog of human genome-wide association studies, was used to identify 1264 SNPs previously found to be associated with BD. BD-associated genes were identified based on the gene nearest to each SNP. The top ten SNPs were identified based on the EMBL-EBI GWAS catalog-reported p-values. 


\section{RegulomeDB analysis}

RegulomeDB [3] analysis was completed by entering the top ten SNPs (by p-value) into the RegulomeDB tool. Rank and probability scores were used to determine the likelihood of each SNP influencing a genetic regulatory element. The RegulomeDB Probability Score ranged from 0 to 1 and referred to the probability of the SNP being a variant. The RegulomeDB Rank ranged from 1a to 7 and it referred to the amount of evidence available for a variant to be located in a functional region, with 1a having the most evidence. In total, the data consisted of the genes, SNPs of each gene, and the probability scores and probability rankings of each SNP, allowing for a meta-analysis of these significant genes and SNPs.

\section{DAVID pathway analysis}

The genes nearest to BD-associated SNPs were used to analyze biological pathways using the Database for Annotation, Visualization and Integrated Discovery (DAVID) [4]. Specifically, after analyzing the genes and SNPs given by EMBL-EBI GWAS, DAVID provided a list of pathways that the specific genes fall into. Based on the analysis of the P-values, percentage of BDassociated genes in the pathway, as well as by building upon the foundation from the SNPs data, an understanding of how each pathway plays a role on $\mathrm{BD}$ were made.

\section{CONCLUSION}

Complex genetic diseases like bipolar disorder are caused by the contribution of many different variants, genes, and pathways, combined with environmental influences. To identify novel genes and biological functions involved in BD, we performed a thorough analysis of SNPs previously associated with disease. We found several SNPs with known links to mental disorders, and SNPs that have not been extensively studied and could be promising targets for future studies. Several of the top SNPs are predicted to fall within gene regulatory elements, suggesting crucial functions of those SNPs in regulating genes involved are involved with bipolar disorder. Moreover, analysis of biological pathways encompassing genes nearest the top SNPs, showed enrichment of alternative splicing, which can result in undesired variation of gene expression and faulty genomic instruction in functional protein, leading to multiple physiological diseases. These results highlight both known and novel biological mechanisms that should be included as targets for future investigation.

\section{Alternative Splicing}

Our results found that the first most significant pathway related to BD-associated genes is alternative splicing. Alternative splicing is the process by which mRNA can be stitched together in different ways such that multiple protein isoforms can be encoded by a single gene. SNPs can impact alternative splicing, resulting in unintended variation in gene expression [10]. Previous research has linked splicing variants with psychiatric disorders [11]. Others have suggested that alternatively spliced genes could be utilized as novel biomarkers for BD [14]. Through further focusing on alternative splicing and its risk variants, new therapeutic treatments can be discovered for bipolar disorder and related mental illnesses.

Synaptic Function Pathways

Our results found that the second most significant pathway related to BD-associated genes was postsynaptic density, which is essential for regulating developmental and structural aspects of the nervous system. Genes in this pathway function to localize glutamate receptors and position neurotransmitter receptors [15]. We also identified the dendritic spine pathway, which is related to the small protrusions from neuronal dendrites that form the post-synaptic component of most excitatory synapses in the brain. Glutamatergic excitatory synapses along this pathway are responsible for the maintenance of these synapses as they play an important role in plasticity [15].

The mechanism of action between ion channels, specifically lithium and calcium are significant. It was found that such ions can stimulate various receptors and neurotransmitters; this can account for the balance of connection between excitatory synapses, neuronal cells, and generally throughout the nervous system -- as well as modulating glutamate / synapses. When this balance is essentially destroyed, this can lead to Bipolar Disorder as certain neurotransmitters and signaling enzymes can lead to limitations of synaptic transmission.

As for bipolar disorder, the genes involved in this pathway include FXR1, DGKI, SHANK2 and MOB4. FXR1: The FXR1 genes have been implicated to cause dysregulation of dopamine receptor signaling. Since dopamine receptors signaling is one of the main known causes of BD, FXR1 theoretically plays a main role. Furthermore, the DGKI gene has shown high correlation with bipolar disorder, as the $\eta$ isozyme of diacylglycerol kinase (DGK) is highly expressed in the hippocampus and Purkinje cells in the central nervous system. Recently, several genome-wide association studies have implicated DGK $\eta$ in the etiology of bipolar disorder (BD).

\section{Golgi Organization}

The Golgi organization pathway has not been connected to Bipolar Disorder in any prior research making this connection a novel finding. The Golgi organization pathway was found to include many of the BD-linked genes, including ATP8B3, CLASP1, LMAN2L, MYO28A, PRKD1, SYNE1, and ASAP2. The Golgi is an organelle that is directly involved in the packaging of proteins into vesicles before transportation. ASAP2 is known to activate small GTPases, including ARF1, regulate the formation of post-Golgi 
vesicles and modulate constitutive secretion. This connection should be explored further as this variant plays a huge role in bipolar disorder.

\section{Relationships with other Neurological Disorders}

Our results from the DAVID functional analysis tool found that bipolar disorder shares several pathways and genes with schizophrenia, narcolepsy, Parkinson's disease, schizophrenia, and major depressive disorder. In recent years, researchers have investigated the relationship between narcolepsy and bipolar disorder which report conflicting results. The AKAP6 gene functions in the sarcoplasmic reticulum of skeletal muscle cells and is a candidate loci for narcolepsy [13]. The involvement of genes which function in skeletal muscle cells in bipolar disorder coupled with the genetic/symptomatic associations with narcolepsy suggest another possible shared pathophysiology between the two disorders which may warrant further investigation. Parkinson's disease has been previously connected to mitochondrial dysfunction and the PRKN gene is known to cause mitochondrial impairment. Mitochondrial impairment will result in apoptosis if the mitochondrial impairment is at a significantly severe level. Mitochondrial dysfunction is an important part of the organelle organization pathway, which is a significant pathway in BD. However, more valuable research is to be done within these sectors to fully defend these conclusions made. The intracellular signal transduction is a significant pathway in bipolar disorder, connecting the cell surface to the nucleus, and targeting many hormones altering hormonal levels for many mood disorders. Kinase-mediated signaling events are important in schizophrenia as imbalances in kinase activity caused by intracellular signaling contributes to many of the observed deficits in schizophrenia.

The integration of genome wide expression or EMBL-EBI GWAS data with pathways and networks is very promising, but it can be improved by considering other information, such as epigenetics. However, the field is still far from maturity due to incomplete pathway knowledge. Furthermore, pathway analysis is currently coding gene-centered; non-protein coding elements (noncoding RNA, non-transcribed regions and epigenetic marks) have not been sufficiently integrated in the analysis. Moreover, research can be done on a larger sample of relevant SNPs, as a contrast to just the top 10 SNPs, to gain a more accurate understanding of these factors. These topics also warrant further, more holistic, research that investigates pathways and networks in relation to bipolar disorder, with the integration of novel factors such as non-protein coding elements, epigenetics, and more thorough analysis of pathways. All these findings show great promise to lead to future research in bipolar disorder and its relation to pathways and genome wide expression.

There is difficulty in understanding disease risk associated with non coding SNPs, and EMBL-EBI GWAS-identified SNPs, as they only serve as representatives for all SNPs in the same haplotype block. SNPs often do not cause changes in protein function but cause changes in gene expression instead. Unlike cystic fibrosis that is caused by mutations in the coding region of a gene, most disease associated SNPs are located in noncoding regions of the genome, which is why we focused on genetic pathways in our paper. Furthermore, SNPs cannot be used to find specific genes that affect the disease, as SNPs often affect many genes.

\section{ACKNOWLEDGMENT}

Thank you to Postdoctoral Scientist at AbbVie Genomics Research Center, Lindsay Stolzenburg, for thorough guidance throughout our work research and more.

Thank you to Nicholas Canaday Spitzer, Professor in the Division of Biological Sciences at the University of California, San Diego for guidance through our research.

\section{REFERENCES}

1. Alonso, J., Petukhova, M., Vilagut, G., Chatterji, S., Heeringa, S., Ustun, T., \& Alhamzaw, A. (2010). Days out of role due to common physical and mental conditions: results from the WHO World Mental Health Surveys. Molecular Psychiatry, 1-13.

2. Grande, I., Berk, M., Birmaher, B., \& Vieta, E. (2015). Bipolar disorder. The Lancet. doi:10.1016/S0140-6736(15)00241-X

3. Alda M. Bipolar disorder: from families to genes. Can J Psychiatry. 1997 May;42(4):378-87. doi: 10.1177/070674379704200404. PMID: 9161762.

4. McGuffin P, Rijsdijk F, Andrew M, Sham P, Katz R, Cardno A. The heritability of bipolar affective disorder and the genetic relationship to unipolar depression. Arch Gen Psychiatry. 2003 May;60(5):497-502. doi: 10.1001/archpsyc.60.5.497. PMID: 12742871.

5. National Institute of Mental Health. (2018, October). bipolar disorder. https://www.nimh.nih.gov/health/publications/bipolar-disorder/index.shtml

6. Nurnberger, J. I., Jr, Koller, D. L., Jung, J., Edenberg, H. J., Foroud, T., Guella, I., Vawter, M. P., Kelsoe, J. R., \& Psychiatric Genomics Consortium Bipolar Group (2014). Identification of pathways for bipolar disorder: a meta-analysis. JAMA psychiatry, 71(6), 657-664. https://doi.org/10.1001/jamapsychiatry.2014.176

7. Isozaki, T., Komenoi, S., Lu, Q., Usuki, T., Tomokata, S., Matsutomo, D., Sakai, H., Bando, K., Kiyonari, H., \& Sakane, F. (2016). Deficiency of diacylglycerol kinase $\eta$ induces lithium-sensitive mania-like behavior. Journal of neurochemistry, 138(3), 448-456. https://doi.org/10.1111/jnc.13661

8. Glatt, S. J., Chandler, S. D., Bousman, C. A., Chana, G., Lucero, G. R., Tatro, E., May, T., Lohr, J. B., Kremen, W. S., Everall, I. P., \& Tsuang, M. T. (2009). Alternatively Spliced Genes as Biomarkers for Schizophrenia, bipolar disorder and Psychosis: A Blood-Based Spliceosome-Profiling Exploratory Study. Current pharmacogenomics and personalized medicine, 7(3), 164-188. https://doi.org/10.2174/1875692110907030164

9. Atz, M. E., Rollins, B., \& Vawter, M. P. (2007). NCAM1 association study of bipolar disorder and schizophrenia: polymorphisms and alternatively spliced isoforms lead to similarities and differences. Psychiatric genetics, 17(2), 55-67. https://doi.org/10.1097/YPG.0b013e328012d850

10. Ishikawa, M., Mizukami, K., Iwakiri, M., \& Asada, T. (2007). Immunohistochemical and immunoblot analysis of Dopamine and cyclic AMP-regulated phosphoprotein, relative molecular mass 32,000 (DARPP-32) in the prefrontal cortex of subjects with schizophrenia and bipolar disorder. Progress in neuropsychopharmacology \& biological psychiatry, 31(6), 1177-1181. https://doi.org/10.1016/j.pnpbp.2007.04.013

11. Hamdani, N., Bengoufa, D., Godin, O., Doukhan, R., Le Guen, E., Daban-Huard, C., Bennabi, M., Delavest, M., Lépine, J. P., Boukouaci, W., Laouamri, H., Houenou, J., Jamain, S., Richard, J. R., Lecorvosier, P., Yolken, R., Rajagopal, K., Leboyer, M., \& Tamouza, R. (2018). Immunoglobulin sub-class distribution in bipolar disorder and schizophrenia: potential relationship with latent Toxoplasma Gondii infection. BMC psychiatry, 18(1), 239. https://doi.org/10.1186/s12888-018-1821-9 
12. Itokawa, M., Yamada, K., Iwayama-Shigeno, Y., Ishitsuka, Y., Detera-Wadleigh, S., \& Yoshikawa, T. (2003). Genetic analysis of a functional GRIN2A promoter (GT)n repeat in bipolar disorder pedigrees in humans. Neuroscience letters, 345(1), 53-56. https://doi.org/10.1016/s0304-

13. D'Onofrio, S., Mahaffey, S., \& Garcia-Rill, E. (2017). Role of calcium channels in bipolar disorder. Current psychopharmacology, 6(2), 122-135. https://doi.org/10.2174/2211556006666171024141949

14. Zeleznikow-Johnston, A. M., Renoir, T., Churilov, L., Li, S., Burrows, E. L., \& Hannan, A. J. (2018). Touchscreen testing reveals clinically relevant cognitive abnormalities in a mouse model of schizophrenia lacking metabotropic glutamate receptor 5. Scientific reports, 8(1), 16412. https://doi.org/10.1038/s41598-018-33929-3

15. Harrison, P.J., Hall, N., Mould, A. et al. Cellular calcium in bipolar disorder: systematic review and meta-analysis. Mol Psychiatry (2019). https://doi.org/10.1038/s41380-019-0622-y

16. Du, J., Quiroz, J., Yuan, P., Zarate, C., \& Manji, H. K. (2004). Bipolar disorder: involvement of signaling cascades and AMPA receptor trafficking at synapses. Neuron glia biology, 1(3), 231-243. https://doi.org/10.1017/S1740925X05000098

17. Szczepankiewicz A. (2013). Evidence for single nucleotide polymorphisms and their association with bipolar disorder. Neuropsychiatric disease and treatment, 9, 1573-1582. https://doi.org/10.2147/NDT.S28117

18. Calabrese, B., Wilson, M. S., \&amp; Halpain, S. (2006). Development and Regulation of Dendritic Spine Synapses. Physiology, 21(1), 38-47. doi:10.1152/physiol.00042.2005

19. Öngür, D., Bechtholt, A. J., Carlezon, W. A., Jr, \& Cohen, B. M. (2014). Glial abnormalities in mood disorders. Harvard review of psychiatry, 22(6), 334337. https://doi.org/10.1097/HRP.0000000000000060

20. Miladinovic, T., Nashed, M. G., \& Singh, G. (2015). Overview of Glutamatergic Dysregulation in Central Pathologies. Biomolecules, 5(4), 3112-3141. https://doi.org/10.3390/biom5043112

21. Singh, V., Erady, C., \& Balasubramanian, N. (2018). Cell-matrix adhesion controls Golgi organization and function through Arf1 activation in anchoragedependent cells. Journal of cell science, 131(16), jcs215855. https://doi.org/10.1242/jcs.215855

22. Corvin A. P. (2010). Neuronal cell adhesion genes: Key players in risk for schizophrenia, bipolar disorder and other neurodevelopmental brain disorders? Cell adhesion \& migration, 4(4), 511-514. https://doi.org/10.4161/cam.4.4.12460

23. Ruoff, C. M., Reaven, N. L., Funk, S. E., McGaughey, K. J., Ohayon, M. M., Guilleminault, C., \& Black, J. (2017). High Rates of Psychiatric Comorbidity in Narcolepsy: Findings From the Burden of Narcolepsy Disease (BOND) Study of 9,312 Patients in the United States. The Journal of clinical psychiatry, 78(2), 171-176. https://doi.org/10.4088/JCP.15m10262

24. Morse, A. M., \& Sanjeev, K. (2018). Narcolepsy and Psychiatric Disorders: Comorbidities or Shared Pathophysiology?. Medical sciences (Basel, Switzerland), 6(1), 16. https://doi.org/10.3390/medsci6010016

25. Larsen, S. B., Hanss, Z., \& Krüger, R. (2018, July). The genetic architecture of mitochondrial dysfunction in Parkinson's disease. Retrieved from https://www.ncbi.nlm.nih.gov/pmc/articles/PMC6015629/

26. Matsumoto-Taniura, N., Pirollet, F., Monroe, R., Gerace, L., \& Westendorf, J. M. (1996). Identification of novel M phase phosphoproteins by expression cloning. Molecular biology of the cell, 7(9), 1455-1469. https://doi.org/10.1091/mbc.7.9.1455

27. Database, G. H. (n.d.). MPHOSPH9 Gene (Protein Coding). Retrieved December 20, 2020, from https://www.genecards.org/cgibin/carddisp.pl?gene=MPHOSPH9\#summaries

28. Varghese, Femina P., and E Sherwood Brown. "The Hypothalamic-Pituitary-Adrenal Axis in Major Depressive Disorder: A Brief Primer for Primary Care Physicians." Primary Care Companion to the Journal of Clinical Psychiatry, Physicians Postgraduate Press, Inc., Aug. 2001, www.ncbi.nlm.nih.gov/pmc/articles/PMC181180/.

29. Miladinovic, T., Nashed, M. G., \& Singh, G. (2015). Overview of Glutamatergic Dysregulation in Central Pathologies. Biomolecules, 5(4), 3112-3141 https://doi.org/10.3390/biom5043112

30. Cooper, G. M. (2000). Signal Transduction and the Cytoskeleton. The Cell: A Molecular Approach. 2nd Edition. https://www.ncbi.nlm.nih.gov/books/NBK9964/

\section{AUTHORS}

Varshene Sivaprakash: varshene15@gmail.com

Ulain Umar: hoorulainumar1@gmail.com

Trisha Thontepu: sandiegotrisha@gmail.com

Rehan Shah: rehanssah12@gmail.com

Karina Anders: kmanders317@gmail.com

Jennifer Xu: jenniferxu50@gmail.com

Jaylen Lockhart: JaylenSydneyLockhart@gmail.com

Gasser Mamdouh: gasser45123@gmail.com

Deeksha Yelamanchi: deeksha.yelamanchi04@gmail.com

Arya Nalavade: aryanalavade04@ gmail.com

Aliza Lopez: ali2312lopez@gmail.com 